\title{
Implementation of normal incidence absorption coefficient for boundary condition of digital Huygens' model
}

\author{
Takao Tsuchiya* \\ Department of Information Systems Design, Doshisha University, \\ 1-3 Miyakodani, Tatara, Kyotanabe, 610-0321 Japan
}

(Received 12 March 2008, Accepted for publication 24 March 2008)

Keywords: Digital Huygens' model (DHM), Boundary condition, Normal incidence absorption coefficient, IIR filter PACS number: $43.55 . \mathrm{Ka}$ [doi:10.1250/ast.29.326]

\section{Introduction}

Time domain numerical analyses of sound fields such as the Finite Difference Time Domain (FDTD) method have become familiar as a result of the recent progress in computing environments. For accurate numerical analysis of sound fields in rooms, it is necessary to model the boundary condition, which has absorption coefficient with an arbitrary frequency characteristics. The surface impedance model is widely used as a boundary condition [1,2] in the FDTD method. Another model has been proposed in which the boundary condition is physically modeled [3]. However, it is difficult to represent the arbitrary frequency characteristics of the absorption coefficient in these models because the boundary conditions are approximately represented by physical models and the number of degrees of freedom for representation of the characteristics is insufficient.

In this paper, an IIR digital filter is applied to the boundary condition of the digital Huygens' model (DHM) in order to implement a normal incidence absorption coefficient with an arbitrary frequency characteristics. The DHM is a physical model in which the propagation and scattering of waves are simulated as sequences of impulses scattered in accordance with Huygens' principle [4-6]. It is easy to implement a digital filter into the boundary of the DHM since the DHM is essentially expressed by a digital equivalent circuit. An IIR filter is connected to the boundary of a DHM network to represent the normal incidence absorption coefficient of the boundary with an arbitrary frequency dependence. In this study, some numerical demonstrations are carried out for one-dimensional sound propagation in air.

\section{Digital Huygens' model (DHM)}

In the DHM, a small three-dimensional acoustic field can be described by a cubic element consisting of six transmission lines, as shown in Fig. 1. The characteristic feature of the DHM is that the sequences of the impulses are traced in the time domain. When an input pulse $P$ is applied to line 1 at the time $t=n \Delta t$, where $n=1,2, \ldots, \Delta t=\Delta l / c_{0}$ and $c_{0}$ is the sound speed, the pulse is scattered at the node because of the impedance discontinuity at the connecting node. The impedance from one line to the other five lines at the node is $Z_{0} / 5$, where $Z_{0}$ is the characteristic impedance of the line, because the five lines are connected in parallel at the node. The reflection coefficient $\Gamma$ at the node is given by

$$
\Gamma=\frac{Z_{0} / 5-Z_{0}}{Z_{0} / 5+Z_{0}}=-\frac{2}{3} .
$$

Thus an impulse with amplitude $-2 / 3 P$ is reflected back to incident line 1 and impulses with amplitude $P / 3$ are scattered to the other five lines. The scattering matrix is thus given as [4]

$$
\left[\begin{array}{c}
S_{1}(n+1) \\
S_{2}(n+1) \\
S_{3}(n+1) \\
S_{4}(n+1) \\
S_{5}(n+1) \\
S_{6}(n+1)
\end{array}\right]=\frac{1}{3}\left[\begin{array}{cccccc}
-2 & 1 & 1 & 1 & 1 & 1 \\
1 & -2 & 1 & 1 & 1 & 1 \\
1 & 1 & -2 & 1 & 1 & 1 \\
1 & 1 & 1 & -2 & 1 & 1 \\
1 & 1 & 1 & 1 & -2 & 1 \\
1 & 1 & 1 & 1 & 1 & -2
\end{array}\right]\left[\begin{array}{c}
P_{1}(n) \\
P_{2}(n) \\
P_{3}(n) \\
P_{4}(n) \\
P_{5}(n) \\
P_{6}(n)
\end{array}\right],
$$

where $P$ and $S$ are incident and scattered pulses, respectively, and subscripts indicate the number of the line. The scattered pulses then become the input pulses to the adjacent elements. The sequence in this process creates the propagation of waves corresponding to Huygens' principle, as the field consists of connected elements forming a network.

\section{Modeling of boundary condition}

In the DHM, the boundary condition can be introduced by the reflection coefficient between the transmission line and the boundary because sound pressure and particle velocity do not explicitly appear in the formulation. The reflection coefficient $r_{0}$ can be calculated from the normal incidence absorption coefficient $\alpha_{0}$ as follows:

*e-mail: ttsuchiy@mail.doshisha.ac.jp 


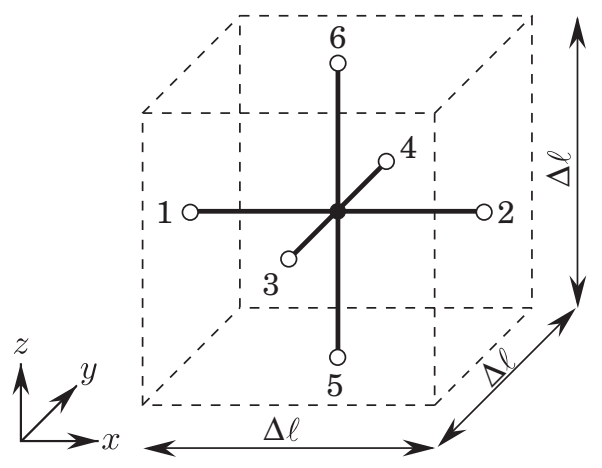

Fig. 1 Three-dimensional DHM element.
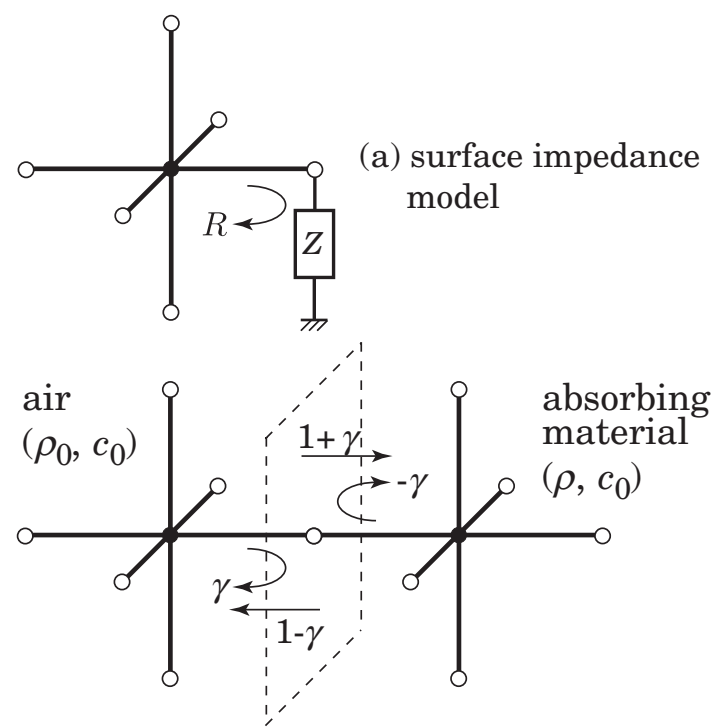

(b) absorbing material model

Fig. 2 Modeling of boundary condition.

$$
r_{0}=\sqrt{1-\alpha_{0}}
$$

There are two models for the boundary condition of the DHM used to implement the normal incidence absorption coefficient, as shown in Fig. 2: one is the surface impedance model and the other is the absorbing material model. In the surface impedance model, the end of the transmission line is terminated by an impedance $Z$ corresponding to the surface acoustic impedance of the boundary, as shown in Fig. 2(a). The pulse is reflected back from the connecting node because of the impedance discontinuity between the line and the impedance. The local reflection coefficient $R$ between the line and the impedance is given as

$$
R=\frac{Z-Z_{0}}{Z+Z_{0}} .
$$

However, the local reflection coefficient $R$ is not equal to the reflection coefficient for the wave reflected at the boundary of the DHM network because the propagation speed on the DHM network is $1 / \sqrt{3}$ of the speed along a single transmission line. For a propagating wave on the DHM network, the reflection coefficient $r$ is therefore represented as

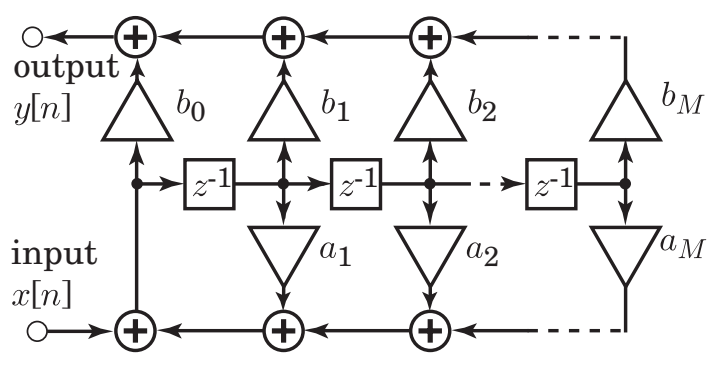

Fig. 3 IIR filter.

$$
r=\frac{Z-Z_{0} / \sqrt{3}}{Z+Z_{0} / \sqrt{3}} .
$$

From Eqs. (4) and (5), we obtain the relation between $R$ and $r$ as [7]

$$
R=\frac{(1+r)-\sqrt{3}(1-r)}{(1+r)+\sqrt{3}(1-r)} .
$$

Thus, to achieve the reflection coefficient $r_{0}$ in Eq. (3) for the propagating wave on the DHM network, the local reflection coefficient $R_{0}$ for the line is

$$
R_{0}=\frac{\left(1+r_{0}\right)-\sqrt{3}\left(1-r_{0}\right)}{\left(1+r_{0}\right)+\sqrt{3}\left(1-r_{0}\right)}
$$

However the local reflection coefficient $R_{0}$ becomes negative when $\alpha_{0} \geq 0.928$ and this makes the filter design difficult.

To avoid the negative reflection coefficient, the absorbing material model can be introduced, as shown in Fig. 2(b). In the absorbing material model, the reflection coefficient $r$ can be calculated from the medium density as

$$
r=\frac{\rho-\rho_{0}}{\rho+\rho_{0}},
$$

where $\rho_{0}$ is the density of air and $\rho$ is the density of absorbing material. In this case, Eq. (8) is directly applied to the DHM calculation because the sound speed does not appear in the equation. In the DHM, the reflection and transmission due to the density discontinuity are respectively introduced using the forced reflection and transmission coefficients at the interface between two media, as illustrated in Fig. 2(b).

In order to implement the frequency characteristics, the reflection coefficient $r$ at the medium interface is changed according to the frequency characteristics of the normal incidence absorption coefficient $\alpha_{0}$. To represent arbitrary frequency characteristics, an IIR filter is connected to the boundary of a DHM network, as shown in Fig. 3. The filter coefficients $a$ and $b$ corresponding to the frequency characteristics of the normal incidence absorption coefficient are calculated by the least P-norm optimal design method provided by the MATLAB filter design toolbox [8].

\section{Numerical experiments}

To verify the validity of the present method, numerical examinations are carried out for one-dimensional sound propagation in air. The numerical model is shown in Fig. 4. The length of the one-dimensional field is $50 \mathrm{~m}$. One end of the field is driven by a velocity with a Gaussian shaped waveform and the other end is connected to an absorbing 


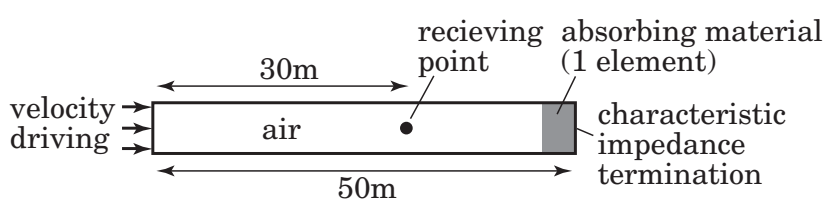

Fig. 4 Numerical model.

Table 1 Filter coefficients of the IIR filter.

\begin{tabular}{lrr}
\hline & Glass wool & Perforated plate \\
\hline$a_{1}$ & -3.674460 & -2.827991 \\
$a_{2}$ & 5.153324 & 2.850796 \\
$a_{3}$ & -3.275843 & -1.199757 \\
$a_{4}$ & 0.802495 & 0.182191 \\
$a_{5}$ & -0.005153 & -0.004450 \\
$b_{0}$ & -0.039948 & 0.847891 \\
$b_{1}$ & 0.104864 & -2.493116 \\
$b_{2}$ & -0.092307 & 2.602006 \\
$b_{3}$ & 0.026274 & -1.133953 \\
$b_{4}$ & 0.000747 & 0.185404 \\
$b_{5}$ & 0.000005 & -0.007423 \\
\hline
\end{tabular}

material in which the normal incidence absorption coefficient has a frequency characteristic. The other end of the absorbing material is terminated by a sound absorber with the characteristic impedance of air. The field is divided into 5,000 DHM elements whose element length is $\Delta l=10 \mathrm{~mm}$. The time step $\Delta t$ is chosen to be $16.8 \mu \mathrm{s}$ and the sound speed $c_{0}$ is 343.7 $\mathrm{m} / \mathrm{s}$. The reflected waveform from the absorbing material is calculated at $x=30 \mathrm{~m}$, then it is compared with that from the rigid wall in the frequency domain. The normal incidence absorption coefficient is calculated from the reflection coefficient at each frequency component. Two absorbing materials are chosen: one is a glass wool whose density is $35 \mathrm{~kg} / \mathrm{m}^{3}$ and thickness is $50 \mathrm{~mm}$, and the other is a perforated plate whose thickness is $7 \mathrm{~mm}$, hole diameter is $6-22 \mathrm{~mm} \phi$ and air cavity depth is $45 \mathrm{~mm}$. The filter coefficients calculated for each material are shown in Table 1. The order of the IIR filter is 6 .

Figures 5 and 6 show the calculated frequency characteristics of the normal incidence absorption coefficient of the glass wool and the perforated plate, respectively. In the figures, the solid lines indicate the result calculated by the DHM with the IIR filter and the dashed lines indicate the desired characteristics. The results calculated by the DHM agree well with the desired results. It is confirmed that the model can be easily applied to absorbing materials with arbitrary frequency characteristics.

\section{Conclusion}

An IIR digital filter is applied to the boundary condition of the digital Huygens' model (DHM) in order to implement a normal incidence absorption coefficient with arbitrary frequency characteristics. The absorbing material model is introduced to implement the IIR filter into the DHM. Some numerical demonstrations are carried out for one-dimensional sound propagation in air. A glass wool and a perforated plate

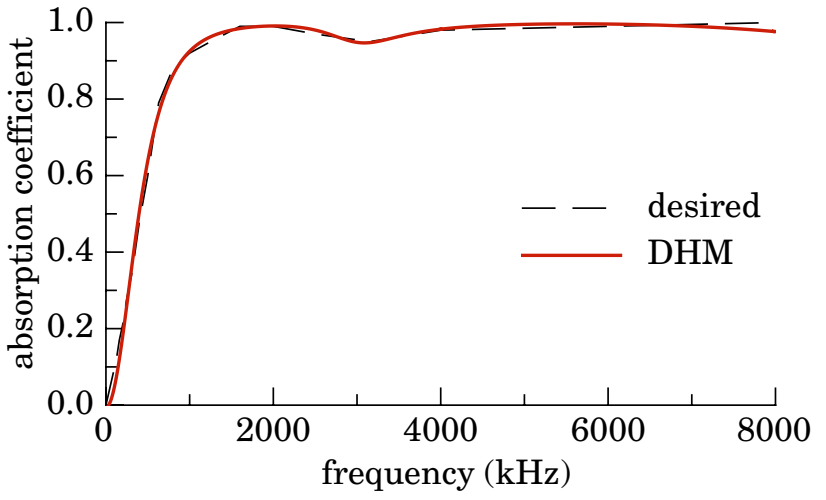

Fig. 5 Normal incidence absorption coefficient calculated for glass wool.

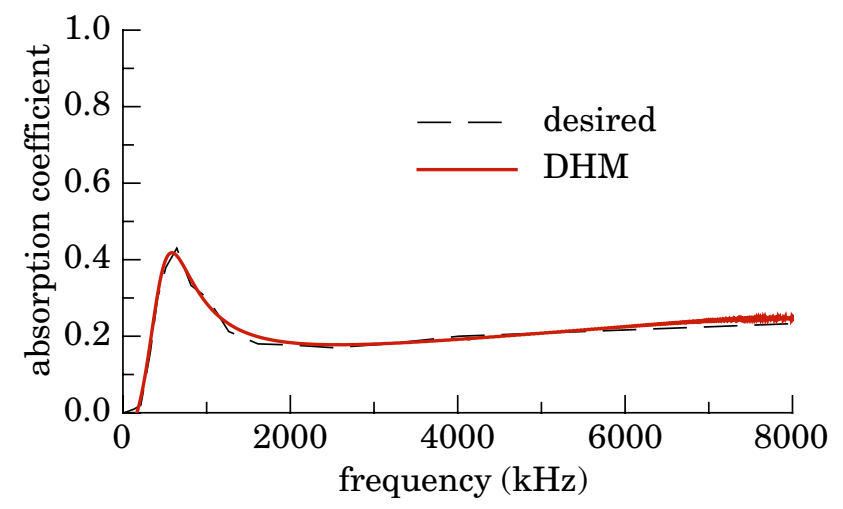

Fig. 6 Normal incidence absorption coefficient calculated for perforated plate.

are chosen as examples of absorbing materials. It is confirmed that the model can be easily applied to absorbing materials with arbitrary frequency characteristics.

\section{References}

[1] O. Chiba, T. Kashiwa, H. Shimoda, S. Kagami and I. Fukai, "Analysis of sound fields in three dimensional space by the time-dependent finite-difference method based on leap frog algorithm," J. Acoust. Soc. Jpn. (J), 49, 551-562 (1993) (in Japanese).

[2] T. Yokota, S. Sakamoto and H. Tachibana, "Visualization of sound propagation and scattering in rooms," Acoust. Sci. \& Tech., 23, 40-46 (2002).

[3] H. Suzuki, A. Omoto and K. Fujiwara, "Treatment of boundary conditions by finite difference time domain method," Acoust. Sci. \& Tech., 28, 16-26 (2007).

[4] Y. Kagawa, T. Tsuchiya, B. Fujii and K. Fujioka, "Discete Huygens' model approach to sound wave propagation," $J$. Sound Vib., 218, 419-444 (1998).

[5] T. Tsuchiya and Y. Kagawa, "Digital equivalent circuits for acoustic field based on discrete Huygens' modeling," Jpn. J. Appl. Phys., 44(6B), 4297-4300 (2005).

[6] T. Tsuchiya, "Numerical simulation of sound wave propagation with sound absorption using digital Huygens' model," Jpn. J. Appl. Phys., 46(7B), 4809-4812 (2007).

[7] Y. Kagawa, N. Yoshida, T. Tsuchiya and M. Sato, Touka Kairomou Hou Nyuumon (Introduction to Equivalent Circuit Network Modeling) (Morikita, Tokyo, 1999) (in Japanese).

[8] Signal Processing Toolbox User's Guide (MathWorks, 2002). 